\title{
A Rare and Fatal Case of Aortobronchial Fistula Secondary to Disseminated Tuberculous Aortitis and Takayasu Arteritis
}

Bryan Rene F. Toledano M.D, Senior Adult Cardiology Fellow in training, ${ }^{1}$ Glenn Valloso M.D, Pathology Resident -5 th year, ${ }^{1}$ Ma. Vanessa Yu M.D, Adult Cardiology - Vascular Research Fellow, ${ }^{1}$ Therese Eileen B. Lladoc-Natividad M.D, Consultant- Internal Medicine, Adult Rheumatology, ${ }_{1}^{1}$ Paul P. Salandanan M.D, Consultant- Internal Medicine, Infectious Diseases, ${ }^{1}$ Leonisa S. Sagun, Consultant- Anatomic and Clinical Pathology, Cardiovascular, ${ }^{1}$ Teresita De Guia M.D, Consultant- Internal Medicine, Adult Pulmonology, ${ }^{1}$ Joel S. Paz M.D, Consultant - Adult Cardiology, Vascular ${ }^{1}$

${ }^{1}$ Philippine Heart Center

Main Author: Bryan Rene F. Toledano M.D

Email Address: imbryantoledano@gmail.com

Contact Number: 09054776260

\begin{abstract}
\section{BACKGROUND}

Aortobronchial fistula (ABF) is a rare complication of a chronic thoracic aneurysm. An ABF secondary to Disseminated Tuberculous (TB) Aortitis and Takayasu Arteritis (TA) may present with extensive combinations of aneurysms and stenoses in different parts of the arterial system with involvement of other organs.
\end{abstract}

\section{CASE PRESENTATION}

A 29-year-old Filipino female, married, who was admitted to our institution due to sudden massive hemoptysis. A Computed Tomography of the chest showed pulmonary tuberculosis with lymph node involvement and pseudoaneurysm of the descending aorta. The pertinent physical examinations are blood pressure (BP) differential of 20mmhg of upper extremities, painless multiple nodular tongue, multiple cervical, and supraclavicular lymphadenopathies, grade $3 / 6$ holosystolic murmur. On Computed Tomography of the aorta, there are combinations of aneurysms of the mid to distal descending thoracic aorta, distal abdominal aorta, superior mesenteric artery with stenoses of the subclavian and left renal arteries. She was immediately started on anti-TB medications, corticosteroids and was scheduled for aortic aneurysmal repair. However, there was a recurrence of massive hemoptysis leading to her demise. An autopsy of the thorax and abdomen revealed an acute pulmonary hemorrhage, aortobronchial fistula secondary to Disseminated Tuberculous Aortitis and Takayasu Arteritis.

\section{CONCLUSION}

A masked ABF should be suspected in a patient with massive hemoptysis and a pseudoaneurysm of the descending aorta. In a young female with disseminated TB aortitis, a concomitant TA should be investigated. Both can share the same clinical, radiological and pathologic features. It is fatal and needs prompt surgical or endovascular intervention.

\begin{abstract}
BACKGROUND
Aortobronchial fistula $(\mathrm{ABF})$ is a rare complication of a chronic thoracic aneurysm. It occurs due to longstanding inflammation with subsequent erosion and formation of a connection between the descending aorta and pulmonary parenchyma or bronchial tree. ${ }^{1}$ The reported etiologies are atherosclerosis and prior aortic surgical intervention in the elderly ${ }^{2}$ while infectious or inflammatory disease in the young. ${ }^{3}$
\end{abstract}

\section{CAse pResentation}

A 29-year-old Filipino female, married, unemployed who was admitted to our institution due to sudden massive hemoptysis. She was brought to a local hospital and was given tranexamic acid 500mg/IV every 8 hours and butamirate 50mg/tab 1 tab every 8 hours which provided a resolution of symptoms. Her blood examinations showed mild anemia hemoglobin $115 \mathrm{~g} / \mathrm{L}$, hematocrit 0.35, platelet 396 109/L, white blood cell count 7.9, neutrophils, $75 \%$ lymphocyte $17 \%$, normal prothrombin time $100 \%$ activity, INR 1.00 , slightly elevated activated prothrombin time of 38.8 secs (24.8-38.3) and normal serum creatinine 0.05 $\mathrm{mmol} / \mathrm{L}$. On chest $\mathrm{x}$-ray, a reticulonodular opacities in the right upper lobe and convex opacity in the left retrocardiac region were seen. A Computed Tomography of the chest was done which showed lobulated aneurysm of the descending aorta hence she was referred at our institution. At the emergency room, the patient was conscious, coherent, not in respiratory distress. Her vital signs and anthropometric measurements were BP 110/80 mm Hg right, 130/80 left upper extremities, HR 67 bpm, RR 20 cpm, T 36.8, O2 sat 97\% at room air Height $157.8 \mathrm{~cm}$ Weight $40 \mathrm{~kg}$ and BMl $16.9 \mathrm{~kg} / \mathrm{m}^{2}$. She has pale palpebral conjunctiva, anicteric sclera, painless multiple nodular tongue nonulcerative and multiple cervical, supraclavicular lymphadenopathies. There is symmetrical chest expansion, vesicular breath sounds, dynamic precordium, apex beat at 5 th left intercostal space, midclavicular line, grade $3 / 6$ holosystolic murmur at the left lower sternal border and pulse is $1+$ for the upper extremity. 
On further workup, 12-lead electrocardiogram showed sinus rhythm, Two-dimensional echocardiography with doppler showed a small Ventricular Septal Defect Supracristal type $0.5 \mathrm{~cm}$ with left to right shunt, no vegetations noted and Computed Tomography of the aorta revealed a myriad of findings [Figure 1-6]. Tuberculous aortitis and Takayasu arteritis were considered. She was immediately started on anti-TB medications of Rifampicin 150mg/tab + Isoniazid 75mg/tab + Pyrazinamide $400 \mathrm{mg} / \mathrm{tab}+$ Ethambutol $\mathrm{HCl} 275 \mathrm{mg} / \mathrm{tab} 3$ tablets once a day,
Hydrocortisone 100mg/IV twice a day and was scheduled for aortic aneurysmal debridement and repair. However, on the 3rd hospital day, she had a recurrence of massive hemoptysis and epistaxis accompanied by dyspnea and sudden cardiac arrest. An ACLS was done with no return of spontaneous circulation for 30 minutes. An autopsy of the thorax and abdomen revealed the following gross findings [Figure: 7-9]. The pertinent microscopic findings are the following [Figure 10-15]
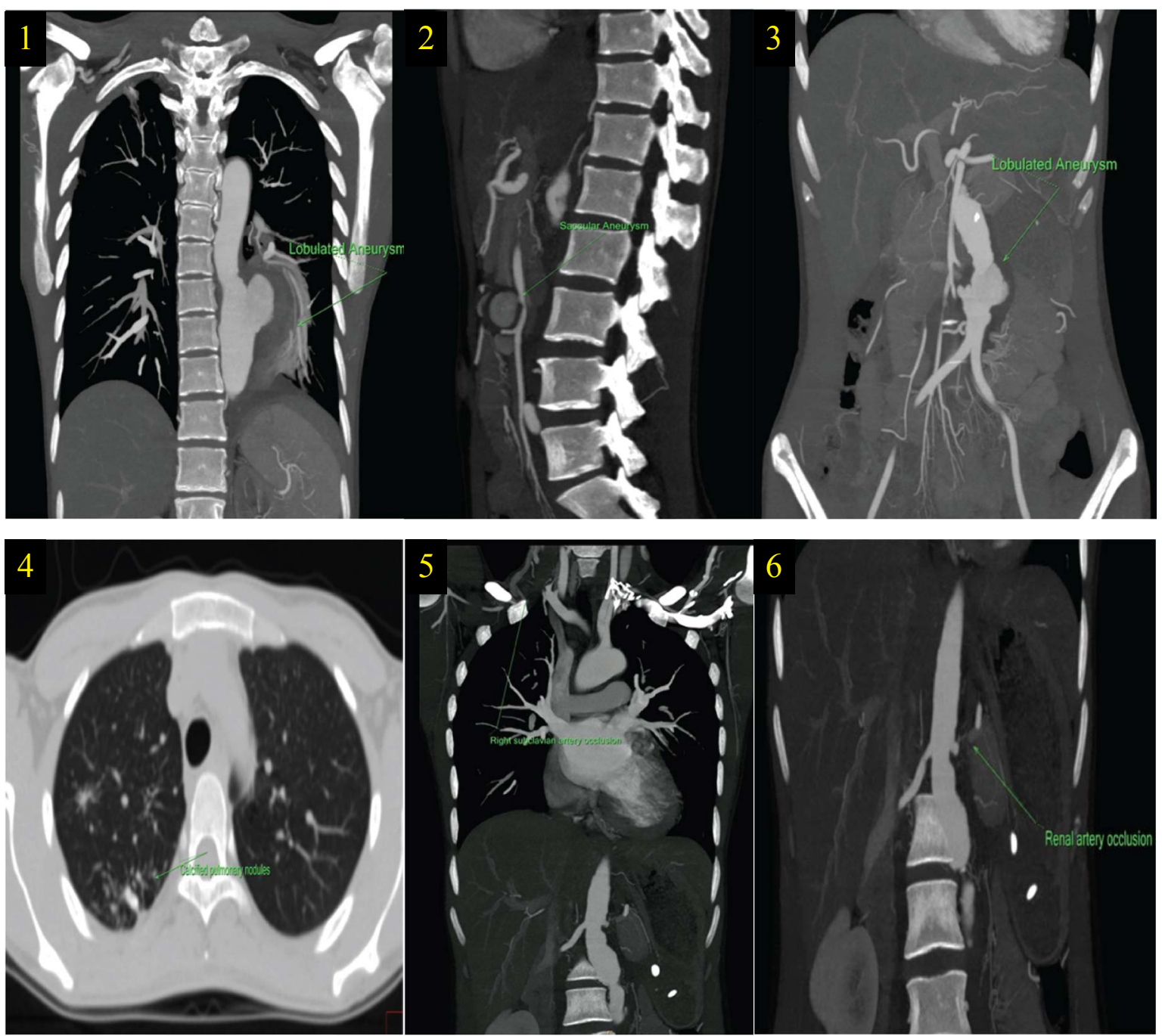

Computed Tomography of the Chest and Aorta: Figure 1. Coronal view image shows lobulated mass from the posterolateral wall of the mid to distal descending thoracic aorta with eccentric thrombus. Figure 2. Sagittal view image shows saccular aneurysm arising from the anterior wall of the distal abdominal aorta, with eccentric thrombus .Figure 3. Coronal view image shows lobulated aneurysm arising from the anterior wall of the superior mesenteric artery just after the take-off of the jejunal branch. Figure 4. Coronal view image shows non-opacified right subclavian artery due to occlusion. Figure 5. Coronal view image shows occluded mid segment of the left renal artery with small-sized kidney and delayed enhancement. Figure 6. Axial view image shows calcified nodules with surrounding pleuroparenchymal fibrosis and tiny centrilobular nodules in tree bud pattern. 

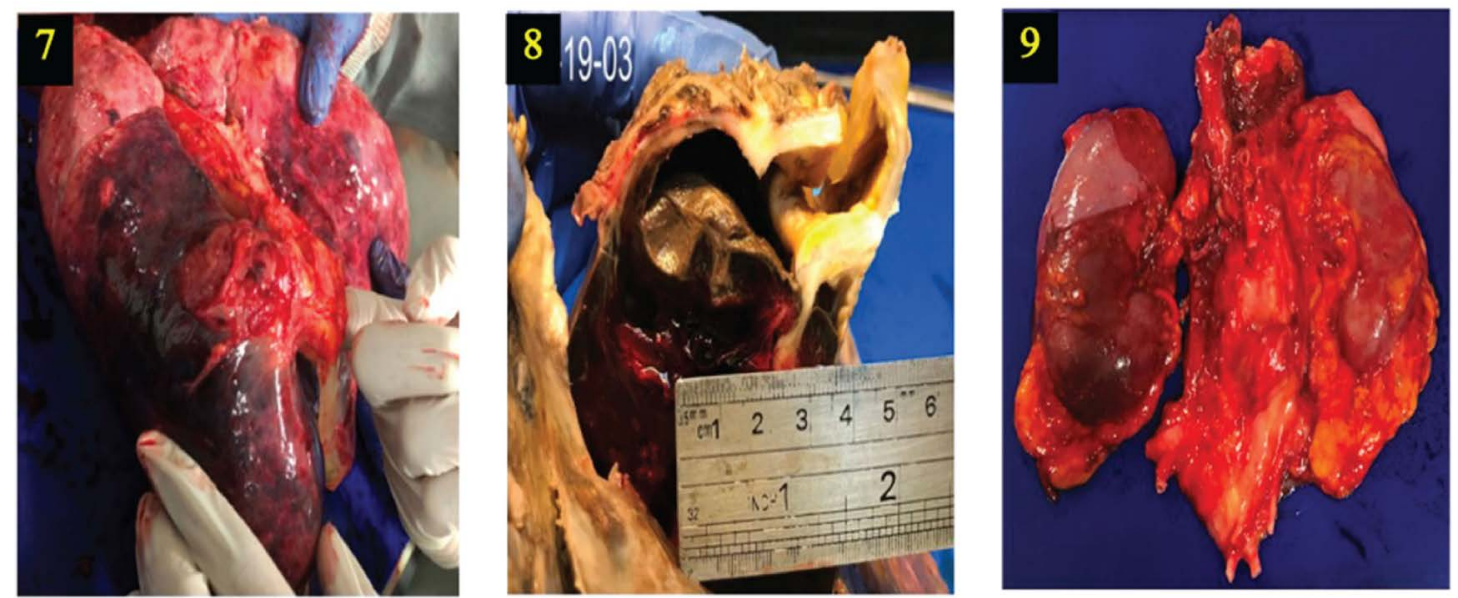

Figure 7. Posterior image shows massively hemorrhagic lung parenchyma, the middle and distal descending aneurysmal thoracic aorta is closely adherent to the left lower lobe. Figure 8. Fistulous tract in between measuring $1.5 \mathrm{~cm}$ in diameter and $3.5 \mathrm{~cm}$ in length. Figure 9. Anterior image shows the left kidney is smaller than the right with a lobulated aneurysm seen in the distal abdominal aorta.
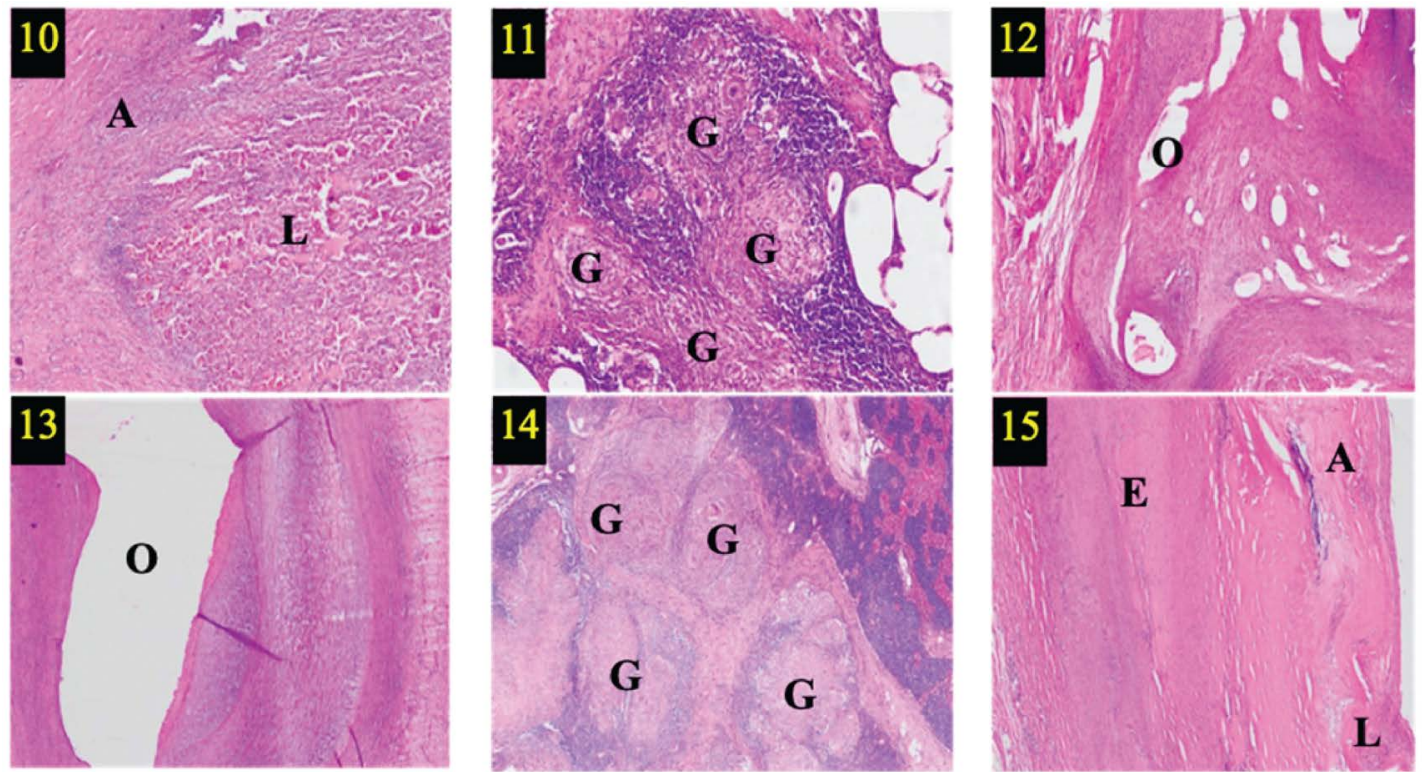

Figure 10. H\&E stain 40x magnification image shows sections of the fistula with aneurysmal wall tissue(A) breaching the hemorrhagic pulmonary parenchyma(L) with scattered inflammatory cells. Figure 11. H\&E stain 100x magnification image shows sections from the right upper lobe with variable-sized noncaseating granulomas $(\mathrm{G})$, some are vaguely caseating, composed of cohesive clusters of histiocytes, usually with admixed lymphocytes, eosinophils, neutrophils, multinucleated giant cells, and epithelioid cells. Figure 12. H\&E stain 40x magnification image shows the right subclavian artery with $80-90 \%$ atherosclerotic occlusion $(O)$ with neovascularization and moderate disruption of the elastic lamina and fibrosis. Figure 13. H\&E stain 40x magnification image shows the left subclavian artery with $25-35 \%$ atherosclerotic occlusion $(0)$ and moderate disruption of the elastic lamina and fibrosis. Figure 14. H\&E stain 40x magnification image shows the lymph node with follicular hyperplasia, prominence of postcapillary venules, increased number of immunoblasts, plasma cells and histiocytes and fibrosis; there are well defined multiple granulomas $(\mathrm{G})$, variable caseating necrosis, and Langerhans giant cells. Figure 15. H\&E stain 100x magnification image shows the aneurysmal thoracic aorta with atherosclerosis(A), lymphoplasmacytic infiltrates $(\mathrm{L})$, and moderate disruption of the elastic lamina and fibrosis(E). 


\section{DISCUSSION}

The extent of involvement of the vascular system with combinations of aneurysms of the mid to distal descending thoracic aorta, distal abdominal aorta, superior mesenteric artery, stenoses of the subclavian and left renal arteries have lead to Tuberculous aortitis and Takayasu arteritis as the primary considerations. A TA was suspected and subsequently diagnosed based on the American College of Rheumatology criteria of age $<40$ years old, blood pressure difference $>10 \mathrm{mmhg}$ and arteriogram narrowing of the subclavian and renal artery. The presence of these 3 criteria yields a sensitivity of $91 \%$ and specificity of $98 \% .{ }^{4}$ The coexistence of Disseminated Tuberculous aortitis and Takayasu arteritis have been documented by retrospective studies and several case reports. The pathogenesis is linked to the heightened response of TA patients from the mycobacterium antigens particularly the 65 kDa Heat shock protein. ${ }^{5}$ The microscopic findings are consistent with chronic vasculitis and chronic granulomatous inflammation suggestive of TA and TB. Both entities may show replacement fibrosis of the adventia, destruction of elastic lamina, infiltration of lymphocytes and obliterative endarteritis during their late stages ${ }^{6}$ as seen in our patient.

\section{CONCLUSION}

A masked ABF should be suspected in a patient with massive hemoptysis and a pseudoaneurysm of the descending aorta. In a young female with disseminated TB aortitis, a concomitant TA should be investigated. Both can share the same clinical, radiological and pathologic features. It is fatal and needs prompt surgical or endovascular intervention.

\section{KEYWORDS}

Aortobronchial fistula, Takayasu arteritis, TB aortitis

\section{REFERENCES}

1. Posacioglu $\mathrm{H}$, and Apaydin Anil, Pseudoaneurym and Aortobronchial fistula after coarctation repair by patch aortoplasty. Texas Heart Ins. 31:319-2, 2004

2. Liu SF, Chen YC, Lin MC, and Kao CL, Thoracic aortic aneurysm with aortobronchial fistula: thirteen-year experience. Heart and Lung, The Journal of Cardiopulmonary and Acute care. Vol 33 Issue 2: 119-123, 2004.

3. Deipolyi AR, Czaplicki CD, and Oklu Rhami, Inflammatory and Infectious aortic disease, Cardiovascular Diagnostic and Therapy. S61-70, 2018.

4. Imamura R, Hayashi K, Sada KE, Yamamura Y, Yamaguchi S, Morishita M, Watanabe $\mathrm{H}$, Matsumoto $\mathrm{Y}$, and Wada $\mathrm{Y}$, Hemoptysis originating from the bronchial artery in Takayasu arteritis with Ulcerative Colitis, The Japanese Society of Internal Medicine. 1463-18, 2018.

5. Aggarwal A, Chag M, Sinha N, and Naik S. Takayasu's arteritis: role of Mycobacterium tuberculosis and its $6 \mathrm{kDa}$ heat shock protein, International Journal of Cardiology. Vol 55 Issue 1,5: 49-55, 1996.

6. Johnston SL, Lock RJ, and Gompels MM. Takayasu Arteritis: a review. J. Clinical Pathol. 55:481-486, 2002. 